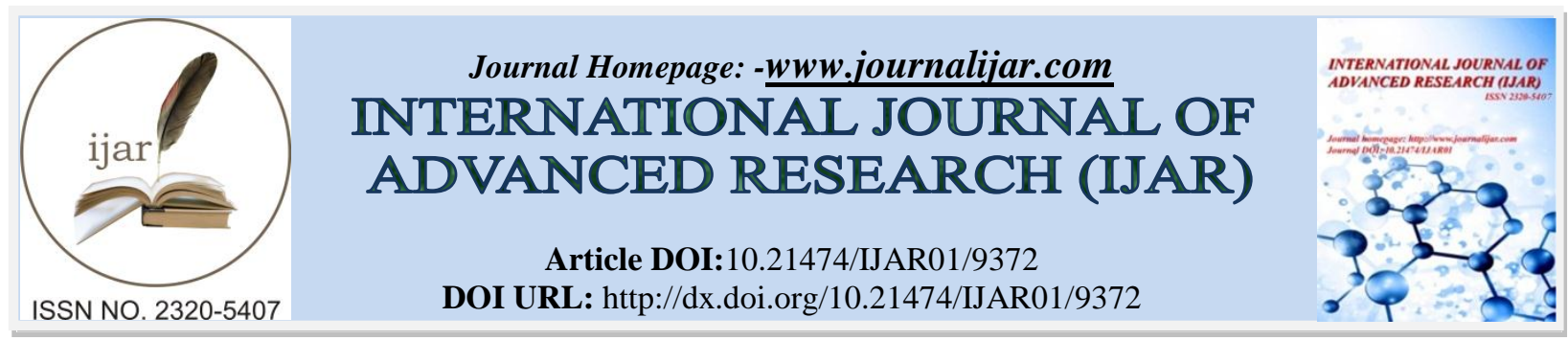

RESEARCH ARTICLE

\title{
A STUDY ON PREVALENCE OF MYTHS RELATED TO DENTAL HEALTH CARE AMONG THE POPULATION OF DISTRICT LUCKNOW.
}

\author{
Bhaskar Agarwal ${ }^{1}$, M K Agarwal ${ }^{2}$, Srishti Goel Agarwal ${ }^{3}$, Manoj Pandey ${ }^{4}$, Kiran Tiwari ${ }^{5}$ and Sunit Kumar \\ Jurel $^{6}$.
}

1. Associate Professor, King George's Medical University, Lucknow.

2. Director, Dr. G. L. Gupta Institute of Public Health, University of Lucknow, Lucknow.

3. Director, Dental O3, Lucknow.

4. Assistant Professor, CB Gupta BSS Mahavidhayalaya, Lucknow.

5. Junior Engineer, Irrigation Department, Lucknow.

6. Associate Professor, King George's Medical University, Lucknow.

\section{Manuscript Info}

Manuscript History

Received: 10 May 2019

Final Accepted: 12 June 2019

Published: July 2019

Key words:-

Dental Myths, Public Health, Oral Health, Tobacco.

\section{Abstract}

Introduction: Today we believe that our society is very well aware of dentist, dentistry and its principles, but this crude traveling has its marks left in the minds of our society, and these exist in the form of myths.

Aim \& Objectives: The aim of this study was to find the prevalence of myths related to dental healthcare among the population of district Lucknow. It was done by finding the prevalence of myths related to dental healthcare among Urban and Rural population of district Lucknow and also comparing both the population.

Material \& Method: A total number of 687 individuals were interviewed out of which 408 were male and 279 were female, with a questionnaire containing a set of nine close-ended multiple choice questions, to facilitate data analysis avoid ambiguity.

Results: Among various myths associated with tooth extraction, the myth that tooth extraction 'weakens eyesight' was most common (71.2\%) followed by 'should not be done during rainy season' (68.1\%), 'consumption of certain contraindicated items causes inflammation in gums' (51.7\%), 'restriction on intake of certain food items' (42.9\%), 'affecting hearing' (13.5\%), affecting brain (12.1\%) and causing hair fall $(13.1 \%)$. For each of the items related with tooth extraction a high proportion of respondents (2.9\% to $18.9 \%$ ) did not reflect any opinion. Conclusion: The findings of present study showed that prevalence of dental myths was more common in underprivileged population, particularly women from lower socioeconomic strata. These findings indicated the need for a community initiative. Keeping in view the modifiable nature of myths, mass awareness campaigns using mass media, particularly audio-visual media are recommended apart from holding dental checkup camps in less privileged urban and rural areas. 


\section{Introduction:-}

Today we believe that our society is very well aware of dentist, dentistry and its principles, but this crude traveling has its marks left in the minds of our society, and these exist in the form of myths. It is generally believed that the urban population of a country is much more educated, much civilized, and moreover has a good infrastructure to prevail much better services of health, education and income (work) but the reality is far behind this assumption. There was no relevant data found in literature exclusively for urban population related to dental myths. ${ }^{1-5}$ With this background in mind a study of myths related to dental health care among the population of district Lucknow was conducted in both urban and rural population of Lucknow.

\section{Aim \& Objectives:-}

The aim of this study was to find the prevalence of myths related to dental healthcare among the population of district Lucknow.

1. To find the prevalence of myths related to dental healthcare among Urban population of district Lucknow.

2. To find the prevalence of myths related to dental healthcare among Rural population of district Lucknow.

3. To compare the prevalence of myths related to dental healthcare among both Urban and Rural population of district Lucknow.

\section{Material \& Method:-}

The study was conducted in two parts: First in the central part of the city and Second part in the rural part of the district. A total number of 687 individuals were interviewed out of which 408 were male and 279 were female, with a questionnaire containing a set of nine close-ended multiple choice questions, to facilitate data analysis avoid ambiguity. All the subjects were interviewed at their home. Initially, the subject of dental myths was discussed informally with friends and colleagues. The ideas and thoughts that transpired were carefully noted and transcripted into salient points. A focus group discussion with colleagues and peers was conducted. From this a matrix of items which could have been included in the questionnaire was evolved.

The questionnaire was made in the local Hindi language and later on translated into English. The subjects participating in the study were questioned about the myths related to tooth extraction, oral prophylaxis and tobacco habits. For the subjects who were illiterate, all the questions were verbally presented to them one by one, and the responses were recorded. It was kept in mind that the subjects should not give any response under any pressure or just to pretend ideal infront of investigator.

Age, Sex, Income and Educational status of all the subjects were recorded and the subjects were divided into groups as follows:

Age-

Group A1: 18-37 years, Group A2: 38-57 years, Group A3: 58 and above

Sex-

Group M: Males, Group F: Females

\section{Educational status-}

\section{Group E0:}

Illiterate, Group E1: Educated up to primary level, Group E2: Educated above primary level.

\section{Monthly Income-}

Group I0: No income, Group I1: Up to 3000, Group I2: 3000-5000. Group I3: above 5000.

After the collection of data it was analyzed using SPSS15.0.

Inclusion and Exclusion criteria was defined and followed.

Study design wasCross sectional study

\section{Results:-}

The present study was carried out with an aim to find out the prevalence of different myths related with dental problems and their treatment, For this purpose, a survey was carried out in which a total of 687 subjects were enrolled. Majority of respondents were males (59.4\%). Majority of respondents were from urban areas (53.6\%). only $14.7 \%$ of respondents were illiterate. Maximum number of respondents had per capita monthly income between Rs $3,000-5,000 /-(45.6 \%)$. 
Evaluation of myths was done under three categories:

1. Myths associated with extraction of teeth

2. Myths related with oral prophyalxis

3. Myths associated with tobacco/pan use

\section{Myths Associated with Extraction of Teeth}

A total of eight myths were explored:

1. Extraction of teeth affects eyesight

2. Extraction of teeth affects brain

3. Extraction of teeth affects hearing

4. Extraction of teeth does not affect anything

5. Extraction of teeth should not be done during rainy season

6. Extraction of teeth does not restrict intake of certain food items for certain time

7. Consumption of restricted items might cause inflammation of gums

Among various myths associated with tooth extraction, the myth that tooth extraction 'weakens eyesight' was most common $(71.2 \%)$ (Table 2)

Table 2:-Frequency Table for Myths associated with Tooth Extraction

\begin{tabular}{|c|l|c|c|c|c|c|c|}
\hline \multirow{2}{*}{ SN } & \multicolumn{1}{|c|}{ Myth } & \multicolumn{2}{c|}{$\begin{array}{c}\text { Respondents } \\
\text { holding myth }\end{array}$} & \multicolumn{2}{c|}{$\begin{array}{c}\text { Respondents } \\
\text { denying myths }\end{array}$} & \multicolumn{2}{c|}{ Non-responders } \\
\cline { 3 - 7 } & & No. & \% & No. & \% & No. & \% \\
\hline 1. & Weakens eyesight & 489 & 71.2 & 68 & 9.9 & 130 & 18.9 \\
\hline 2. & Affects brain & 83 & 12.1 & 474 & 69.0 & 130 & 18.9 \\
\hline 3. & Affects hearing & 56 & 13.5 & 464 & 67.5 & 130 & 18.9 \\
\hline 4. & Causes hairfall & 90 & 13.1 & 556 & 86.1 & 41 & 6.0 \\
\hline 5. & Does not affect & 468 & 68.1 & 187 & 27.2 & 32 & 4.7 \\
\hline 6. & $\begin{array}{l}\text { Should not be done during rainy } \\
\text { season }\end{array}$ & 295 & 42.9 & 372 & 54.1 & 20 & 2.9 \\
\hline 7. & $\begin{array}{l}\text { Restricts intake of certain food items } \\
\text { for some days }\end{array}$ & 355 & 51.7 & 308 & 44.8 & 24 & 3.5 \\
\hline 8. & $\begin{array}{l}\text { Consumption of such items causes } \\
\text { inflammation in gums }\end{array}$ & & & & & \\
\hline
\end{tabular}

The myth that oral prophylaxis weakens teeth was widespread as majority of respondents (50.1\%) endorsed it as a myth held by them. (Table 3)

Table 3:-Frequency Table for Myths associated with Oral Prophylaxis and Pan/Tobacco use

\begin{tabular}{|c|l|c|c|c|c|c|c|}
\hline \multirow{2}{*}{ SN } & \multicolumn{1}{|c|}{ Myth } & \multicolumn{2}{c|}{$\begin{array}{c}\text { Respondents } \\
\text { Holding Myth }\end{array}$} & \multicolumn{2}{c|}{$\begin{array}{c}\text { Respondents } \\
\text { Denying Myths }\end{array}$} & \multicolumn{2}{c|}{ Non-Responders } \\
\cline { 3 - 8 } & & No. & \% & No. & \% & No. & \% \\
\hline 1. & Oral prophylaxis weakens teeth & 344 & 50.1 & 323 & 47.0 & 20 & 2.9 \\
\hline 2. & $\begin{array}{l}\text { Tobacco/Pan use reduces risk of } \\
\text { dental caries and periodontitis }\end{array}$ & 164 & 23.9 & 511 & 74.4 & 12 & 1.7 \\
\hline 3. & Tobacco/Pan reduce dental pain & 156 & 22.7 & 515 & 75.0 & 16 & 2.7 \\
\hline
\end{tabular}

A total of $8(1.2 \%)$ respondents were indecisive with total score 0 but were non-responders on 6 or more items. (Table 4)

Table 4:-Categorization of Total Myth Scores

\begin{tabular}{|c|l|c|c|}
\hline SN & \multicolumn{1}{|c|}{ Myth } & No. & \% \\
\hline 1. & Indecisive (Non-responders on 6 or more items) & 8 & 1.2 \\
\hline 2. & Generally progressive (Scores <2) & 191 & 27.8 \\
\hline 3. & Somewhat conservative (Scores 3 and 4) & 183 & 26.6 \\
\hline 4. & Generally conservative (Scores 5 and 6) & 269 & 39.2 \\
\hline 5. & Strongly conservative (Scores 7 and above) & 36 & 5.2 \\
\hline
\end{tabular}


Statistically, the difference between two groups was significant for all the items except item tooth extraction affects hearing. (Table 5a)

Table 5a:-Itemwise association of demographic profile with myths associated with tooth extraction (a) Region

\begin{tabular}{|c|c|c|c|c|c|c|c|}
\hline \multirow[t]{2}{*}{ SN } & \multirow[t]{2}{*}{ Myth } & \multicolumn{2}{|c|}{ Rural (n=319) } & \multicolumn{2}{|c|}{ Urban $(n=368)$} & \multicolumn{2}{|c|}{$\begin{array}{c}\text { Statistical } \\
\text { significance }\end{array}$} \\
\hline & & No. & $\%$ & No. & $\%$ & $\chi^{2}$ & 'p' \\
\hline 1. & Weakens eyesight & 267 & 83.7 & 222 & 60.3 & 45.50 & $<0.001$ \\
\hline 2. & Affects brain & 47 & 14.7 & 36 & 9.8 & 3.943 & 0.047 \\
\hline 3. & Affects hearing & 45 & 14.1 & 48 & 13.0 & 0.165 & 0.685 \\
\hline 4. & Causes hairfall & 16 & 5.0 & 40 & 10.9 & 7.821 & 0.005 \\
\hline 5. & Does not affect & 12 & 3.8 & 78 & 21.2 & 45.82 & $<0.001$ \\
\hline 6. & $\begin{array}{l}\text { Should not be done during rainy } \\
\text { season }\end{array}$ & 192 & 60.2 & 276 & 75.0 & 17.26 & $<0.001$ \\
\hline 7. & $\begin{array}{l}\text { Restricts intake of certain food } \\
\text { items for some days }\end{array}$ & 159 & 49.8 & 136 & 37.0 & 11.58 & $<0.001$ \\
\hline 8. & $\begin{array}{l}\text { Consumption of such items causes } \\
\text { inflammation in gums }\end{array}$ & 199 & 62.4 & 156 & 42.4 & 27.35 & $<0.001$ \\
\hline
\end{tabular}

Statistically, the difference between two genders was also significant for all the items except the item that tooth extraction should not be done during rainy season. (Table 5b)

Table 5b:-Itemwise association of demographic profile with myths associated with tooth extraction

\begin{tabular}{|c|l|c|c|c|c|c|c|}
\hline SN & \multicolumn{1}{|c|}{ Myth } & \multicolumn{2}{c|}{ Male (n=408) } & \multicolumn{2}{c|}{ Female (n=279) } & \multicolumn{2}{c|}{$\begin{array}{c}\text { Statistical } \\
\text { significance }\end{array}$} \\
\cline { 3 - 8 } & & No. & \% & No. & \% & $\chi^{2}$ & 'p' \\
\hline 1. & Weakens eyesight & 274 & 67.2 & 215 & 77.1 & 7.923 & 0.005 \\
\hline 2. & Affects brain & 40 & 9.8 & 43 & 15.4 & 4.906 & 0.027 \\
\hline 3. & Affects hearing & 40 & 9.8 & 53 & 19.0 & 11.962 & 0.001 \\
\hline 4. & Causes hairfall & 20 & 4.9 & 36 & 12.9 & 14.168 & $<0.001$ \\
\hline 5. & Does not affect & 82 & 20.1 & 8 & 2.9 & 43.21 & $<0.001$ \\
\hline 6. & $\begin{array}{l}\text { Should not be done during rainy } \\
\text { season }\end{array}$ & 280 & 68.6 & 188 & 67.4 & 0.118 & 0.731 \\
\hline 7. & $\begin{array}{l}\text { Restricts intake of certain food } \\
\text { items for some days }\end{array}$ & 144 & 35.3 & 151 & 54.1 & 23.972 & $<0.001$ \\
\hline 8. & $\begin{array}{l}\text { Consumption of such items causes } \\
\text { inflammation in gums }\end{array}$ & 180 & 44.1 & 175 & 62.7 & 22.97 & $<0.001$ \\
\hline
\end{tabular}

for myths that teeth extraction affects hearing, causes hair fall and consumption of contraindicated items might cause inflammation in gums were unaffected by the age of patient $(p>0.05)$. (Table $5 c)$

Table 5c:-Itemwise association of demographic profile with myths associated with tooth extraction

\begin{tabular}{|c|c|c|c|c|c|c|c|c|c|}
\hline \multicolumn{10}{|c|}{ (c) Age } \\
\hline \multirow[t]{2}{*}{ SN } & \multirow[t]{2}{*}{ Myth } & \multicolumn{2}{|c|}{$\begin{array}{c}\text { 18-37 Years } \\
(n=409)\end{array}$} & \multicolumn{2}{|c|}{$\begin{array}{l}\text { 38-57 Years } \\
(\mathbf{n}=184)\end{array}$} & \multicolumn{2}{|c|}{$\begin{array}{c}58 \& \text { above } \\
(\mathrm{n}=94)\end{array}$} & \multicolumn{2}{|c|}{$\begin{array}{c}\text { Statistical } \\
\text { significance }\end{array}$} \\
\hline & & No. & $\%$ & No. & $\%$ & No. & $\%$ & $\chi^{2}$ & 'p' \\
\hline 1. & Weakens eyesight & 278 & 68.0 & 145 & 78.8 & 66 & 70.2 & 7.31 & 0.025 \\
\hline 2. & Affects brain & 49 & 12.0 & 22 & 12.0 & 12 & 12.8 & 0.048 & 0.976 \\
\hline 3. & Affects hearing & 44 & 10.8 & 37 & 20.1 & 12 & 12.8 & 9.536 & 0.008 \\
\hline 4. & Causes hairfall & 36 & 8.8 & 12 & 6.5 & 8 & 8.5 & 0.900 & 0.638 \\
\hline 5 . & Does not affect & 43 & 10.5 & 23 & 12.5 & 24 & 25.5 & 15.22 & $<0.001$ \\
\hline 6. & $\begin{array}{l}\text { Should not be done during rainy } \\
\text { season }\end{array}$ & 261 & 63.8 & 130 & 70.7 & 77 & 81.9 & 12.273 & 0.002 \\
\hline 7. & Restricts intake of certain food & 157 & 38.4 & 92 & 50.0 & 46 & 48.9 & 8.584 & 0.014 \\
\hline
\end{tabular}




\begin{tabular}{|c|l|c|c|c|c|c|c|c|c|}
\hline & items for some days & & & & & & & & \\
\hline 8. & $\begin{array}{l}\text { Consumption of such items } \\
\text { causes inflammation in gums }\end{array}$ & 201 & 49.1 & 108 & 58.7 & 46 & 48.9 & 4.963 & 0.084 \\
\hline
\end{tabular}

For items, tooth extraction restricts intake of certain food items for some days and consumption of such items causes inflammation in gums, the frequency of those holding the myths was lower in literates as compared to illiterates $(\mathrm{p}<0.001)$. (Table 5d)

Table 5d:-Itemwise association of demographic profile with myths associated with tooth extraction

(d) Education

\begin{tabular}{|c|c|c|c|c|c|c|c|c|c|}
\hline \multirow[t]{2}{*}{ SN } & \multirow[t]{2}{*}{ Myth } & \multicolumn{2}{|c|}{$\begin{array}{c}\text { Illiterate } \\
(n=101)\end{array}$} & \multicolumn{2}{|c|}{$\begin{array}{l}\text { Upto Primary } \\
\text { level }(n=32)\end{array}$} & \multicolumn{2}{|c|}{$\begin{array}{c}\text { Above } \\
\text { Primary level } \\
(n=554)\end{array}$} & \multicolumn{2}{|c|}{$\begin{array}{c}\text { Statistical } \\
\text { significance }\end{array}$} \\
\hline & & No. & $\%$ & No. & $\%$ & No. & $\%$ & $\chi^{2}$ & 'p' \\
\hline 1. & Weakens eyesight & 89 & 88.1 & 32 & 100.0 & 368 & 66.4 & 33.19 & $<0.001$ \\
\hline 2. & Affects brain & 26 & 25.7 & 0 & 0.0 & 57 & 10.3 & 23.82 & $<0.001$ \\
\hline 3. & Affects hearing & 13 & 12.9 & 0 & 0.0 & 80 & 14.4 & 5.435 & 0.066 \\
\hline 4. & Causes hairfall & 8 & 7.9 & 4 & 12.5 & 44 & 7.9 & 0.848 & 0.654 \\
\hline 5. & Does not affect & 4 & 4.0 & 0 & 0.0 & 86 & 15.5 & 15.09 & 0.001 \\
\hline 6. & $\begin{array}{l}\text { Should not be done during rainy } \\
\text { season }\end{array}$ & 71 & 70.3 & 24 & 75.0 & 373 & 67.3 & 1.078 & 0.583 \\
\hline 7. & $\begin{array}{l}\text { Restricts intake of certain food } \\
\text { items for some days }\end{array}$ & 73 & 72.3 & 12 & 37.5 & 210 & 37.9 & 41.59 & $<0.001$ \\
\hline 8. & $\begin{array}{l}\text { Consumption of such items } \\
\text { causes inflammation in gums }\end{array}$ & 81 & 80.2 & 12 & 37.5 & 262 & 47.3 & 39.74 & $<0.001$ \\
\hline
\end{tabular}

For items weakens eyesight, affects hearing, does not affect general health, restricts intake of certain food items for some days and consumption of restricted items causes inflammation in gums a statistically significant association between income and prevalence of myths was observed. (Table 5e)

Table 5e:-Itemwise association of demographic profile with myths associated with tooth extraction

(e) Income

\begin{tabular}{|c|c|c|c|c|c|c|c|c|c|}
\hline \multirow[t]{2}{*}{ SN } & \multirow[t]{2}{*}{ Myth } & \multicolumn{2}{|c|}{$\leq 3000(n=165)$} & \multicolumn{2}{|c|}{$\begin{array}{c}\text { 3000-5000 } \\
(\mathbf{n}=313)\end{array}$} & \multicolumn{2}{|c|}{$>5000(n=209)$} & \multicolumn{2}{|c|}{$\begin{array}{c}\text { Statistical } \\
\text { significance }\end{array}$} \\
\hline & & No. & $\%$ & No. & $\%$ & No. & $\%$ & $\chi^{2}$ & 'p' \\
\hline 1. & Weakens eyesight & 145 & 87.9 & 210 & 67.1 & 134 & 64.1 & 30.06 & $<0.001$ \\
\hline 2. & Affects brain & 21 & 12.7 & 33 & 10.5 & 29 & 13.9 & 1.395 & 0.498 \\
\hline 3. & Affects hearing & 8 & 4.8 & 49 & 15.7 & 36 & 17.2 & 14.27 & 0.001 \\
\hline 4. & Causes hairfall & 8 & 4.8 & 24 & 7.7 & 24 & 11.5 & 5.601 & 0.051 \\
\hline 5. & Does not affect & 8 & 4.8 & 43 & 13.7 & 39 & 18.7 & 15.565 & $<0.001$ \\
\hline 6. & $\begin{array}{l}\text { Should not be done during rainy } \\
\text { season }\end{array}$ & 103 & 62.4 & 212 & 67.7 & 153 & 73.2 & 4.976 & 0.083 \\
\hline 7. & $\begin{array}{l}\text { Restricts intake of certain food } \\
\text { items for some days }\end{array}$ & 93 & 56.4 & 125 & 39.9 & 77 & 36.8 & 16.459 & $<0.001$ \\
\hline 8. & $\begin{array}{l}\text { Consumption of such items } \\
\text { causes inflammation in gums }\end{array}$ & 117 & 70.9 & 141 & 45.0 & 97 & 46.4 & 32.268 & $<0.001$ \\
\hline
\end{tabular}

For item Tobacco/Pan reduce dental pain, the difference between two regions was not significant. (Table 6a)

Table 6a:-Itemwise association of demographic profile with myths associated with oral prophylaxis and tobacco use

(a) Region

\begin{tabular}{|c|l|c|c|c|c|c|c|}
\hline SN & \multicolumn{2}{|c|}{ Myth } & \multicolumn{2}{c|}{ Rural (n=319) } & \multicolumn{2}{c|}{ Urban (n=368) } & \multicolumn{2}{c|}{$\begin{array}{c}\text { Statistical } \\
\text { significance }\end{array}$} \\
\cline { 3 - 7 } & & No. & \% & No. & \% & $\chi^{\mathbf{2}}$ & 'p' \\
\hline 1. & Oral prophylaxis weakens teeth & 188 & 58.9 & 156 & 42.4 & 18.71 & $<0.001$ \\
\hline 2. & $\begin{array}{l}\text { Tobacco/Pan use reduces risk of } \\
\text { dental caries and periodontitis }\end{array}$ & 64 & 20.1 & 100 & 27.2 & 4.755 & 0.029 \\
\hline
\end{tabular}



$3 . \quad$ Tobacco/Pan reduce dental pain
\begin{tabular}{l|r}
68 & 21.3 \\
\hline
\end{tabular}
88
23.9
0.656
0.418

For item oral prophylaxis weakens teeth, the proportion of females was higher as compared to that of males whereas for remaining two items, the proportion of males was higher as compared to that of females. However, the association was significant statistically only for the item tobacco/pan reduce dental pain only $(\mathrm{p}<0.001)$. (Table 6b)

Table 6b:-Itemwise association of demographic profile with myths associated with oral prophylaxis and tobacco use

(b) Gender

\begin{tabular}{|c|l|c|c|c|c|c|c|}
\hline SN & \multicolumn{1}{|c|}{ Myth } & \multicolumn{2}{c|}{ Male (n=408) } & \multicolumn{2}{c|}{ Female (n=279) } & \multicolumn{2}{c|}{$\begin{array}{c}\text { Statistical } \\
\text { significance }\end{array}$} \\
\cline { 3 - 8 } & & No. & \% & No. & \% & $\boldsymbol{\chi}^{\mathbf{2}}$ & 'p' \\
\hline 1. & Oral prophylaxis weakens teeth & 192 & 47.1 & 152 & 54.5 & 3.659 & 0.056 \\
\hline 2. & $\begin{array}{l}\text { Tobacco/Pan use reduces risk of } \\
\text { dental caries and periodontitis }\end{array}$ & 100 & 24.5 & 64 & 22.9 & 0.225 & 0.635 \\
\hline 3. & Tobacco/Pan reduce dental pain & 112 & 27.5 & 44 & 15.8 & 12.88 & $<0.001$ \\
\hline
\end{tabular}

Statistically, no significant association between age and myths associated with oral prophylaxis was observed. With increasing age the proportion of respondents holding myth that Tobacco/Pan use reduces risk of dental caries and periodontitis and Tobacco/Pan reduce dental pain increased significantly $(\mathrm{p}<0.05)$. (Table 6c)

Table 6c:-Itemwise association of demographic profile with myths associated with oral prophylaxis and tobacco use

\begin{tabular}{|c|c|c|c|c|c|c|c|c|c|}
\hline \multirow[t]{2}{*}{$\mathbf{S N}$} & \multirow[t]{2}{*}{ Myth } & \multicolumn{2}{|c|}{$\begin{array}{c}\text { 18-37 Years } \\
(n=409)\end{array}$} & \multicolumn{2}{|c|}{$\begin{array}{c}\text { 38-57 Years } \\
(n=184)\end{array}$} & \multicolumn{2}{|c|}{$\begin{array}{c}58 \& \text { above } \\
(n=94)\end{array}$} & \multicolumn{2}{|c|}{$\begin{array}{c}\text { Statistical } \\
\text { significance }\end{array}$} \\
\hline & & No. & $\%$ & No. & $\%$ & No. & $\%$ & $\chi^{2}$ & 'p' \\
\hline 1. & Oral prophylaxis weakens teeth & 213 & 52.1 & 93 & 50.5 & 38 & 40.4 & 4.174 & 0.124 \\
\hline 2. & $\begin{array}{l}\text { Tobacco/Pan use reduces risk of } \\
\text { dental caries and periodontitis }\end{array}$ & 84 & 20.5 & 48 & 26.1 & 32 & 34.0 & 8.349 & 0.015 \\
\hline 3. & Tobacco/Pan reduce dental pain & 76 & 18.6 & 44 & 23.9 & 36 & 38.3 & 17.135 & $<0.001$ \\
\hline
\end{tabular}

For item Tobacco/Pan reduce dental pain, the proportion of those holding this myth was significantly higher among illiterates as compared to literates $(\mathrm{p}=0.032)$. (Table 6d)

Table 6d:-Itemwise association of demographic profile with myths associated with oral prophylaxis and tobacco use

(d) Education

\begin{tabular}{|c|c|c|c|c|c|c|c|c|c|}
\hline \multirow[t]{2}{*}{$\mathbf{S N}$} & \multirow[t]{2}{*}{ Myth } & \multicolumn{2}{|c|}{$\begin{array}{l}\text { Illiterate } \\
(\mathbf{n}=\mathbf{1 0 1})\end{array}$} & \multicolumn{2}{|c|}{$\begin{array}{l}\text { Upto Primary } \\
\text { level }(n=32)\end{array}$} & \multicolumn{2}{|c|}{$\begin{array}{c}\text { Above } \\
\text { Primary level } \\
(n=554)\end{array}$} & \multicolumn{2}{|c|}{$\begin{array}{l}\text { Statistical } \\
\text { significance }\end{array}$} \\
\hline & & No. & $\%$ & No. & $\%$ & No. & $\%$ & $\chi^{2}$ & 'p' \\
\hline 1. & Oral prophylaxis weakens teeth & 51 & 50.5 & 20 & 62.5 & 273 & 49.3 & 2.124 & 0.346 \\
\hline 2. & $\begin{array}{l}\text { Tobacco/Pan use reduces risk of } \\
\text { dental caries and periodontitis }\end{array}$ & 28 & 27.7 & 4 & 12.5 & 132 & 23.8 & 3.102 & 0.212 \\
\hline 3. & Tobacco/Pan reduce dental pain & 32 & 31.7 & 4 & 12.5 & 120 & 21.7 & 6.882 & 0.032 \\
\hline
\end{tabular}

Statistically, no significant association between income and prevalence of myths associated with oral prophylaxis and tobacco use was observed. (Table 6e)

Table 6e:-Itemwise association of demographic profile with myths associated with oral prophylaxis and tobacco use

(e) Income

\begin{tabular}{|c|c|c|c|c|c|c|c|c|c|}
\hline \multirow[t]{2}{*}{ SN } & \multirow[t]{2}{*}{ Myth } & \multicolumn{2}{|c|}{$\leq 3000(n=165)$} & \multicolumn{2}{|c|}{$\begin{array}{c}\text { 3000-5000 } \\
(\mathbf{n}=313)\end{array}$} & \multicolumn{2}{|c|}{$>5000(n=209)$} & \multicolumn{2}{|c|}{$\begin{array}{c}\text { Statistical } \\
\text { significance }\end{array}$} \\
\hline & & No. & $\%$ & No. & $\%$ & No. & $\%$ & $\chi^{2}$ & 'p' \\
\hline 1. & Oral prophylaxis weakens teeth & 90 & 54.5 & 157 & 50.2 & 97 & 46.4 & 2.442 & 0.295 \\
\hline 2. & $\begin{array}{l}\text { Tobacco/Pan use reduces risk of } \\
\text { dental caries and periodontitis }\end{array}$ & 44 & 26.7 & 72 & 23.0 & 48 & 23.0 & 0.933 & 0.627 \\
\hline 3. & Tobacco/Pan reduce dental pain & 40 & 24.2 & 72 & 23.0 & 44 & 21.1 & 0.563 & 0.755 \\
\hline
\end{tabular}


Mean myth scores were significantly higher among males as compared to females, among rural as compared to urban, among older as compared to younger, literate as compared to illiterates, among lower income group as compared to higher income groups $(\mathrm{p}<0.05)$. (Table 7$)$

Table 7:-Association between Demographic Profile and Overall Myth scores

\begin{tabular}{|c|c|c|c|c|c|}
\hline SN & Characteristic & Statistic & $\begin{array}{l}\text { Mean } \\
\text { Score }\end{array}$ & SD & 'p' value \\
\hline \multirow[t]{3}{*}{1.} & Gender & & & & \\
\hline & Male & 408 & 3.59 & 1.72 & \multirow[t]{2}{*}{0.001} \\
\hline & Female & 279 & 4.05 & 1.72 & \\
\hline \multirow[t]{3}{*}{2.} & Place of residence & & & & \\
\hline & Rural & 319 & 3.94 & 1.58 & \multirow[t]{2}{*}{0.019} \\
\hline & Urban & 368 & 3.63 & 1.84 & \\
\hline \multirow[t]{4}{*}{3.} & Agegroup & & & & \\
\hline & 18-37 Years & 409 & 3.53 & 1.71 & \multirow[t]{3}{*}{$<0.001$} \\
\hline & 38-57 Years & 184 & 4.10 & 1.63 & \\
\hline & $58 \&$ above & 94 & 4.22 & 1.83 & \\
\hline \multirow[t]{4}{*}{4.} & Education & & & & \\
\hline & Illiterate & 101 & 4.72 & 1.44 & \multirow[t]{3}{*}{$<0.001$} \\
\hline & Upto Class V & 32 & 3.50 & 0.72 & \\
\hline & Above Class V & 554 & 3.62 & 1.77 & \\
\hline \multirow[t]{4}{*}{5.} & Per capita monthly family income & & & & \\
\hline & Upto Rs 3000/- & 165 & 4.10 & 1.43 & \multirow[t]{3}{*}{0.017} \\
\hline & Rs $3000-5000$ & 313 & 3.64 & 1.83 & \\
\hline & $>$ Rs $5,000 /-$ & 209 & 3.77 & 1.76 & \\
\hline
\end{tabular}

\section{Discussion:-}

The present study was planned to understand the prevalence of dental myths in Lucknow population. For this purpose, a survey was carried out in which both rural and urban population was targeted. The sample size was targeted to be large enough to provide scope for inclusion of almost all the demographic traits.

In previous studies, ${ }^{1-3}$ it has been reported that tooth extraction is often associated with patients' false myths regarding extraction of tooth to be a triggering factor to various systemic problems, moreover, patients' attitude towards getting oral prophylaxis and use of tobacco/pan products has also been seen to be affected by some myths which are primarily negative in nature and have an adverse effect on the dental health of individuals.

Hence, we targeted a total of 8 myths associated with tooth extraction, myth associated with oral prophylaxis and two myths associated with tobacco/pan use. The selection of these myths was done from a general consensus building among a pool of fellow dental practitioners and faculty of dental colleges in the city.

Among various myths associated with tooth extraction, the myth that tooth extraction 'weakens eyesight' was most common (71.2\%) followed by 'should not be done during rainy season' $(68.1 \%)$, 'consumption of certain contraindicated items causes inflammation in gums' $(51.7 \%)$, 'restriction on intake of certain food items' (42.9\%), 'affecting hearing' (13.5\%), affecting brain (12.1\%) and causing hair fall $(13.1 \%)$.

These myths often discourage the patients from getting their carious teeth extracted and in turn deteriorate the entire oral health and functionality.

With respect to myths associated with oral prophylaxis and tobacco/pan use too, it was interesting to see that the myth that oral prophylaxis weakens teeth was widespread as majority of respondents (50.1\%). There were 164 $(23.9 \%)$ respondents who were of the view that tobacco/pan use reduces risk of dental caries and periodontitis. The myth that tobacco/pan reduces dental pain was also held by a sizeable proportion of respondents $(22.7 \%) .{ }^{1,3}$ Existence of such myths is a barrier in the path of utilization of dental care and at the same time is a motivation towards promotion of such habits that otherwise have adverse impact on the oral health as well as general health too. 
These findings thus suggested that in Lucknow district, the prevalence of myths is quite high and needs to be addressed seriously in order to take preventive steps to protect the dental health and at the same time to also avoid evolution of such measures which adversely affect the dental and general health of patients.

For this purpose, we evaluated the association of dental myths with demographic profile of the patients in order to identify the target areas where the interventions to bust these myths could be made while at the same time it could also be identified as to what should be the direction of interventional strategy among patients with different demography.

\section{Problem Areas}

As such with respect to conservativeness to carry the dental myths, majority of surveyed population (71.0\%) was categorized as somewhat to strongly conservative with respect to magnitude of myths. These findings indicated that a generalized awareness programme is recommended in view of such widespread existence of dental myths.

\section{Target population for a Proposed Intervention}

All these myths were more common in rural as compared to urban population, in females as compared to males, in older as compared to younger, illiterates as compared to literates and to some extent more common in lower income groups as compared to higher income groups. Thus, the most preferred target population for intervention was identified as rural, poor, illiterate, elderly females.

\section{Direction of Strategy}

Once targeted population and problem areas were identified, the direction of strategy could be outlined as follows:

1. Awareness campaigns

2. Use of mass media

3. Preferably through audio-visual campaigns

4. Social messaging

5. Door to door visit

6. Organization of dental health care camps in less privileged urban and rural areas.

The findings of present study were elaborative yet not exhaustive, their pattern might vary in different geographical areas and among different demographies, hence further studies at multiple centres to assess existence of various dental myths and their manifestation pattern across different demographic groups is recommended.

\section{Conclusion:-}

The findings of present study showed that prevalence of dental myths was more common in underprivileged population, particularly women from lower socioeconomic strata. These findings indicated the need for a community initiative. Keeping in view the modifiable nature of myths, mass awareness campaigns using mass media, particularly audio-visual media are recommended apart from holding dental checkup camps in less privileged urban and rural areas.

\section{Reference:-}

1. Devesh Tewari, L Nagesh and Manish Kumar. Myths Related to Dentistry in the Rural Population of Bareilly District: A Cross-Sectional Survey. Journal of Dental Sciences and Oral Rehabilitation 2014;5(2):58-64.

2. Mythri H, Santhosh Kumar R. Perceived myths about oral health in India. Indian Journal of Dental Research 2015;26(3):333.

3. Singh SV, Tripathi A, Akbar Z, Chandra S, Tripathi A. Prevalence of dental myths, oral hygiene methods and tobacco habits in an ageing North Indian rural population. Gerodontology 2012;29(2):53-56.

4. R Vignesh, Indra Priyadarshni. Assessment of the prevalence of myths regarding oral health among general population in Maduravoyal, Chennai. Journal of education and ethics in dentistry 2012;2(2):85-91.

5. Ramandeep Singh Gambhir, Ashotosh Nirola, Samir Anand, Tarun Gupta. Myths regarding oral health among patients visiting a dental school in North India: A cross-sectional survey. International Journal of oral health sciences 2015;5(1):9-14. 